\title{
Bisdemethoxycurcumin Increases Sirt1 to Antagonize $t$-BHP-Induced Premature Senescence in WI38 Fibroblast Cells
}

\author{
Ying-Bo Li, ${ }^{1,2}$ Zhang-Feng Zhong, ${ }^{1}$ Mei-Wan Chen, ${ }^{1}$ Jiao-Lin Bao, ${ }^{1}$ Guo-Sheng Wu, \\ Qing-Wen Zhang, ${ }^{1}$ Simon Ming-Yuen Lee, ${ }^{1}$ Pui-Man Hoi, ${ }^{1}$ and Yi-Tao Wang ${ }^{1}$ \\ ${ }^{1}$ State Key Laboratory of Quality Research in Chinese Medicine, Institute of Chinese Medical Sciences, University of Macau, \\ Avenue Padre Tomás Pereira S.J., Taipa 999078, Macau \\ ${ }^{2}$ State Key Laboratory of Natural and Biomimetic Drugs, School of Pharmaceutical Sciences, Peking University, \\ Beijing 100191, China
}

Correspondence should be addressed to Pui-Man Hoi; maghoi@umac.mo and Yi-Tao Wang; ytwang@umac.mo

Received 17 April 2013; Accepted 30 July 2013

Academic Editor: Seung-Heon Hong

Copyright ( 2013 Ying-Bo Li et al. This is an open access article distributed under the Creative Commons Attribution License, which permits unrestricted use, distribution, and reproduction in any medium, provided the original work is properly cited.

\begin{abstract}
Curcuminoids are well known for their capabilities to combat risk factors that are associated with ageing and cellular senescence. Recent reports have demonstrated that curcuminoids can extend the lifespan of model organisms. However, the underlying mechanisms by which these polyphenic compounds exert these beneficial effects remain unknown. In this study, $t$-BHPinduced premature senescence model in human fibroblasts was chosen to explore the protective effects of a curcuminoid, bisdemethoxycurcumin (BDMC), on cellular senescence. The results demonstrated that BDMC attenuated oxidative stress-induced senescence-like features which include the induction of an enlarged cellular appearance, higher frequency of senescence-associated $\beta$-galactosidase staining activity, appearance of senescence-associated heterochromatic foci in nuclei, decrease in proliferation capability, and alteration in related molecules such as p16 and retinoblastoma protein. Notably, we found that BDMC treatment activated Sirtl/AMPK signaling pathway. Moreover, downregulating Sirtl by the pharmacological inhibitor nicotianamine or small interfering RNA blocked BDMC-mediated protection against $t$-BHP-mediated decrease in proliferation. These results suggested that BDMC prevented $t$-BHP-induced cellular senescence, and BDMC-induced Sirtl may be a mechanism mediating its beneficial effects.
\end{abstract}

\section{Introduction}

Curcuminoids, including curcumin, demethoxycurcumin, and bisdemethoxycurcumin (BDMC), are the main active components that are derived from the rhizome of Curcuma longa, which is a commonly used spice in curry dishes. Extensive studies have shown that curcuminoids possess a wide spectrum of therapeutic benefits with low toxicity. These include protection against inflammation, carcinogens, and cardiovascular and neurodegenerative diseases. Although these pathologies have individual complex mechanisms, ageing can contribute to the etiology and risk factors related to these age-associated disorders [1-3]. It was demonstrated that curcuminoids have potent antioxidative capability, including scavenging free radicals and enhancing antioxidant defense. Since oxidative stress and oxidative stress-induced damage accumulation are important trigger factors of cellular senescence and ageing $[4,5]$, it is possible that curcuminoids may exert protective roles in the ageing process. Indeed, it has been recently reported that the administration of curcumin could extend the lifespan of two strains of Drosophila melanogaster [6]. In addition, it was demonstrated that feeding with tetrahydrocurcumin, a major metabolite of curcumin, can increase the average lifespan of male C57BL/6 mice [7]. These observations present curcuminoids as attractive candidates in prevention of ageing and ageing-related disorders. However, the molecular mechanisms by which curcuminoids exert their effects remain unknown.

Various studies highlighted the promising ability of polyphenolic compounds to combat risk factors associated with ageing and even to prevent cellular senescence and ageing. It has been shown that resveratrol, a well-studied 
polyphenol in red wine, can protect against oxidative stressinduced apoptosis, promote proliferation and prevent senescence in endothelial cells [8-10]. In addition, dietary administrations of resveratrol, quercetin, or blueberry polyphenols increased lifespan and stress resistance in Caenorhabditis elegans [11-14]. Notably, apart from the inhibitory effect against oxidative insults, modulation on specific molecules such as Sirtl has been implicated as a major contributing factor for these functions of resveratrol and other polyphenolic compounds. Sirtl, a conserved $\mathrm{NAD}^{+}$-dependent deacetylase, is a primary mediator of cellular metabolism and lifespan extension following caloric restriction [15]. Increase in Sir2 (homolog of Sirt1) activity prolonged the lifespan of yeast, worms, and flies $[16,17]$, suggesting that Sirtl plays a crucial role in ageing and ageing-related diseases. Since polyphenolic compounds, such as curcuminoids, have beneficial effects on ageing-associated disorders, this raised the possibility that Sirtl functions as a mediator for the effects of curcuminoids. Therefore, in this study, we explored the effects of BDMC on oxidative stress-induced premature senescence as well as its effects on Sirtl.

\section{Materials and Methods}

2.1. Reagents. Bisdemethyoxycurcumin (BDMC) was separated from Curcuma longa (turmeric). Its structure (Figure 2(d)) was deduced on the basis of their physicochemical properties and spectral data, and the purity of the compound was $>98 \%$. Basal medium Eagle medium (BME), fetal calf serum (FCS), $t$-BHP solution $70 \%$ in $\mathrm{H}_{2} \mathrm{O}$, and 5-bromo-4-chloro-3-indolyl- $\beta$-D-galactopyranoside were supplied by Sigma-Aldrich (USA). MTT [3-(4,5-dimethyl-2thiazolyl)-2,5-diphenyl tetrazolium bromide] was purchased from USB (USA). Hoechst 33342 dye was purchased from Molecular Probe (USA). 4'-6-Diamidino-2-phenylindole (DAPI) was purchased from Millipore (USA). Antibodies against p21 (F-5), p16 (C-20), and Sirtl were purchased from Santa Cruz (USA). Antibodies against phosphor-AMPK, AMPK, phospho-ERK1/2, phospho-Rb, Rb, and $\beta$-actin were purchased from Cell Signaling Technology (USA). Nicotinamide was purchased from Beyotime (China).

2.2. Cell Culture. The WI-38 human diploid fibroblasts (HDFs) were purchased from ATCC (USA). These cells were maintained in $37^{\circ} \mathrm{C}$ and $5 \% \mathrm{CO}_{2}$ in BME supplemented $10 \%$ $(\mathrm{v} / \mathrm{v})$ FCS. The cells have a finite lifetime of 50 (plus or minus 10) population doublings (PD) with a doubling time of 24 hours and are considered to be young earlier than PD30 and fully senescent at PD50 or later.

2.3. Drug Treatment. To induce premature senescence, subconfluent HDFs at early passage were treated five times for $1 \mathrm{~h}$ with $30 \mu \mathrm{M} t$-BHP freshly diluted in BME with $10 \%$ FCS. At the end of each stress, the HDFs were washed with PBS and cultivated in fresh BME medium with 10\% FCS. In the BDMC-treated group, cells were exposed to BDMC at different concentrations for the indicated period before senescence induction. BDMC was dissolved in DMSO and its concentration did not exceed $0.2 \%$ of the total volume.
2.4. Senescence-Associated $\beta$-Galactosidase (SA $\beta$-Gal) Staining. SA $\beta$-galactosidase-positive cells were detected as described previously [18]. HDFs were fixed for 3-5 min at room temperature in 3\% formaldehyde. After being washed with PBS, cells were incubated at $37^{\circ} \mathrm{C}$ without $\mathrm{CO}_{2}$ with fresh SA- $\beta$-Gal staining solution: $1 \mathrm{mg}$ of 5-bromo-4-chloro3 -indolyl- $\beta$-D-galactopyranoside per $\mathrm{mL}$ (stock $=20 \mathrm{mg}$ of dimethylformamide per $\mathrm{mL}$ ) $/ 40 \mathrm{mM}$ citric acid/sodium phosphate, $\mathrm{pH} 6.0 / 5 \mathrm{mM}$ potassium ferrocyamide $/ 5 \mathrm{mM}$ potassium ferricyanide/ $150 \mathrm{mM} \mathrm{NaCl} / 2 \mathrm{mM} \mathrm{MgCl}_{2}$. Cells were examined after $10-16 \mathrm{~h}$ incubation.

\subsection{Senescence-Associated Heterochromatic Foci (SAHF) Ana-} lysis. SAHF was determined as described previously [19]. Cells were fixed with $4 \%$ para formaldehyde followed by permeabilization with $0.1 \%$ Triton X-100 for $10 \mathrm{~min}$. Then, nucleic acid was stained by DAPI $(5 \mu \mathrm{g} / \mathrm{mL})$ for $3 \mathrm{~min}$. After three washes with PBS, stained cells were examined using a fluorescent microscope (Carl Zeiss, Axiovert 200, USA).

2.6. Determination of Cell Proliferation. After drug treatment, HDFs were seeded at $2 \times 10^{3}$ cells/well in 96-well plates and allowed to grow in BME with 10\% FCS for another $72 \mathrm{~h}$. Cells were then incubated with $100 \mu \mathrm{L}$ BME medium containing $0.5 \mathrm{mg} / \mathrm{mL} \mathrm{MTT}$. After $4 \mathrm{~h}$ incubation at $37^{\circ} \mathrm{C}$, cell supernatants were discarded, MTT crystals were dissolved in $100 \mu \mathrm{L}$ DMSO, and absorbance was measured at $570 \mathrm{~nm}$ using a Multilabel counter (Perkin Elmer, 1420 Multilabel Counter Victor3, Wellesley, USA). The absorbance reflects the cell number after proliferation for $72 \mathrm{~h}$ with different treatment procedures.

2.7. Western Blot Analysis. Protein was extracted using RIPA lysis buffer with $1 \%$ phenylmethanesulfonyl fluoride and $1 \%$ protease inhibitor. Lysates were centrifuged at $12,000 \times \mathrm{g}$ for $20 \mathrm{~min}$ at $4^{\circ} \mathrm{C}$, and the supernatant was collected. Total protein concentrations were determined using BCA Protein Assay kit. Supernatants containing $40 \mu \mathrm{g}$ of protein/lane were separated by SDS-PAGE. After electrophoresis, the separated proteins were electrically transferred onto polyvinylidene difluoride membranes. The membrane was probed with a primary antibody followed by a second antibody and visualized using an ECL advanced Western blotting detection kit. Photos of protein bands were taken by Molecular Imager ChemiDoc XRS (Biorad, USA). Densitometric measurements of band intensity in the Western blots were performed using Quantity One Software.

2.8. Reverse Transcription and Real-Time PCR. Total RNA was isolated using Trizol reagent (Invitrogen, USA). Firststrand cDNA were generated from RNA samples by reverse transcription using oligo (dT), followed by real-time PCR by LightCycler 480 Real-time PCR instrument (Roche, USA). The flowering primers were used to amplify fragments of the human Sirtl: $5^{\prime}$-GACTTCAGGTCAAGGGATGGT$3^{\prime}(\mathrm{F})$ and $5^{\prime}$-CAGCGTGTCTATGTTCTGGGTAT- $3^{\prime}$ (R). GAPDH: $5^{\prime}$-CATGAGAAGTATGACCAACAGCCT-3' (F) and $5^{\prime}$-AGTCCTTCCACGATACCAAAGT-3' (R). 


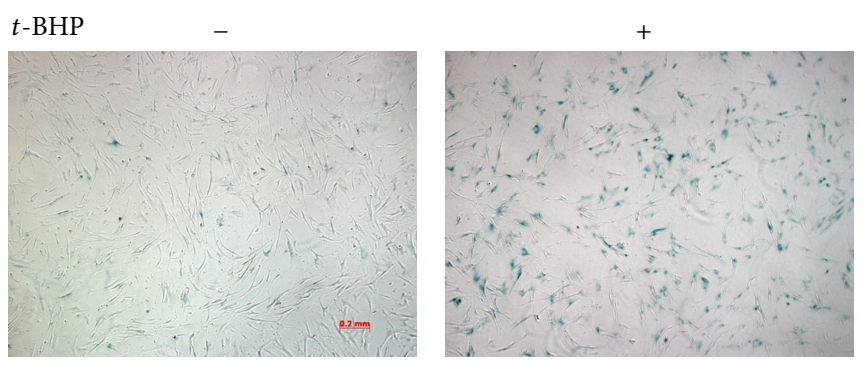

Early passage

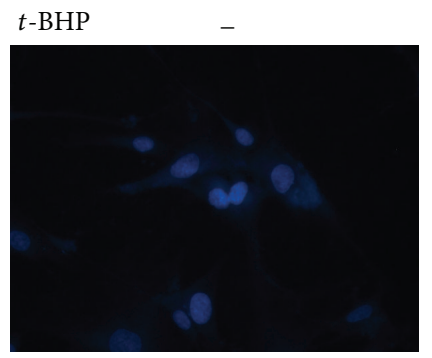

Early passage

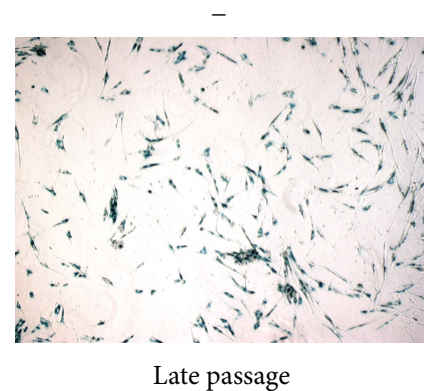

Late passage

(a)
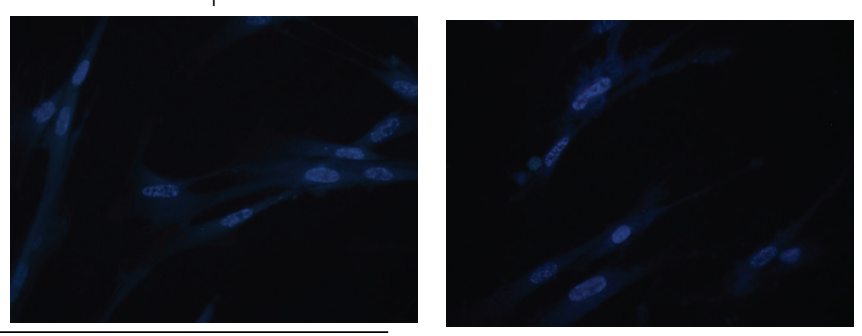

Late passage

(b)

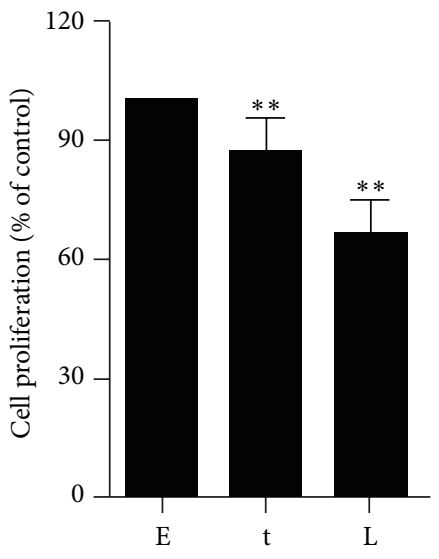

(c)

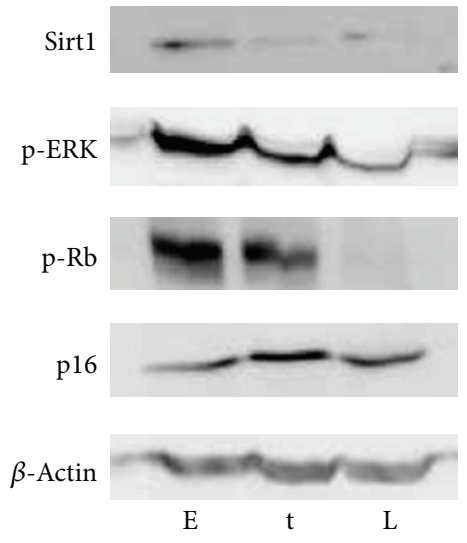

(d)

FIGURE 1: $t$-BHP treatment induced changes of senescence-associated features in WI38 cells. WI38 cells at early passage were treated by $t$ BHP to induce premature senescence; then SA $\beta$-gal staining (a), DAPI staining (b), cell proliferation assay (c), or Western blot assay using specific antibodies (d) were performed in early passage cells (E), $t$-BHP-induced premature senescent cells $(\mathrm{t})$, and replicative senescent cells $(\mathrm{L})$, respectively. Data were presented as mean $\pm \mathrm{SD}$ of three independent experiments. ${ }^{* *} P<0.01$ compared with control group.

2.9. Short Interfering Ribonucleic Acid Transfection. The short interfering ribonucleic acid (siRNA) duplexes used in this study were chemically synthesized by GenePharma Corporation (Shanghai, China). 5'-CGGGAAUCCAAAGGAUAAUTT- $3^{\prime}$ and $5^{\prime}$-AUUAUCCUUUGGAUUCCCGTT- $3^{\prime}$ or $5^{\prime}$ GUAUGACAACAGCCUCAAGTT- $3^{\prime}$ and $5^{\prime}$-CUUGAGGCUGUUGUCAUACTT-3' were used to repress Sirtl or GADPH expression, respectively. Nonsilencing siRNA ( $5^{\prime}$ UUCUCCGAACGUGUCACGUTT- $3^{\prime}$ and $5^{\prime}$-ACGUGACACGUUCGGAGAATT- $3^{\prime}$ ) was used as a negative control. siRNA duplexes were transfected into WI38 HDFs with Lipofectamine 2000 transfection reagent (Invitrogen, USA) according to the manufacturer's instructions.
2.10. Statistical Analysis. All results were presented as mean \pm SD of three independent experiments. Data were analyzed by Student's $t$-test after analysis of variance. $P<0.05$ was considered to be statistically significant.

\section{Results}

3.1. t-BHP Treatment Induced Changes of Senescence-Associated Features in WI38 HDFs. It was well accepted that longterm treatment with sublethal oxidative stresses can induce cells to senescent-like growth arrest, and it therefore has been frequently used for investigating cellular alteration due to oxidative stress-induced premature senescence [20-23]. 

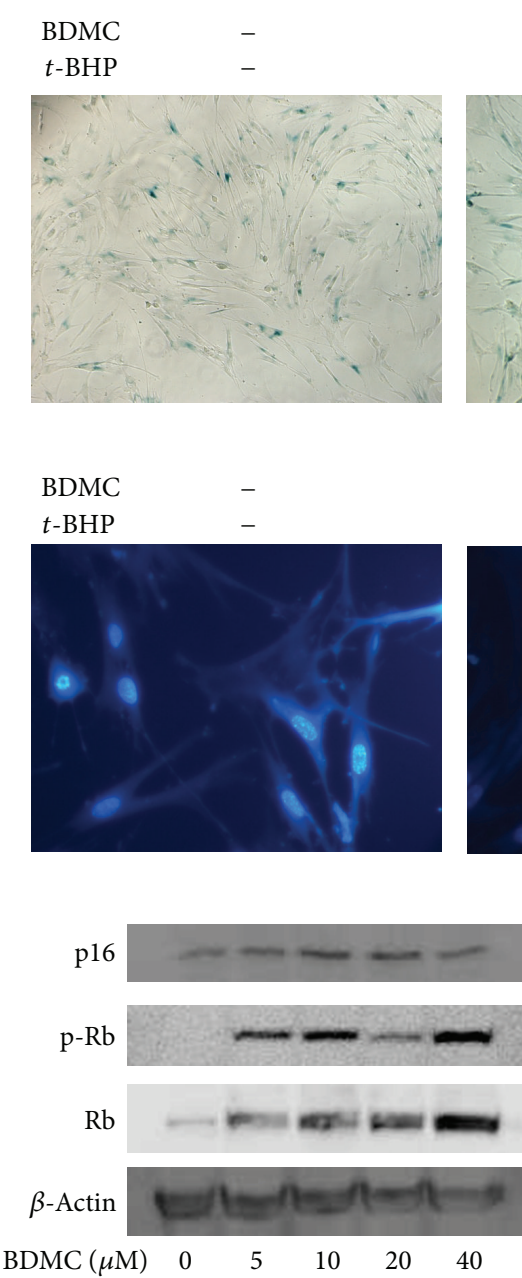

(c)

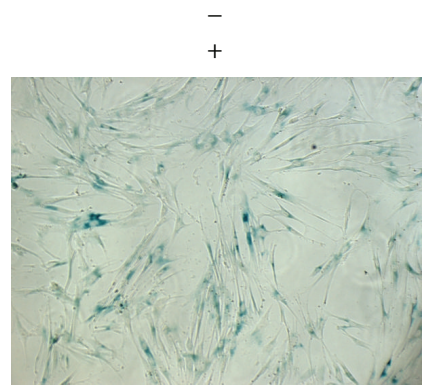

(a)
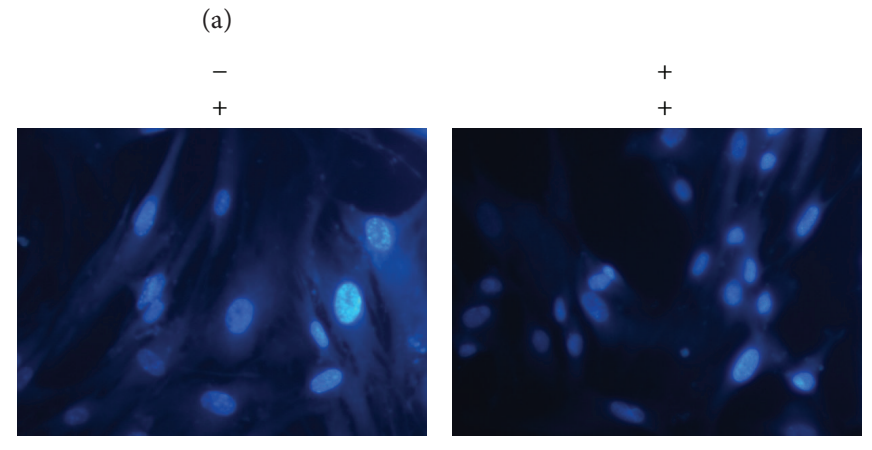

(b)

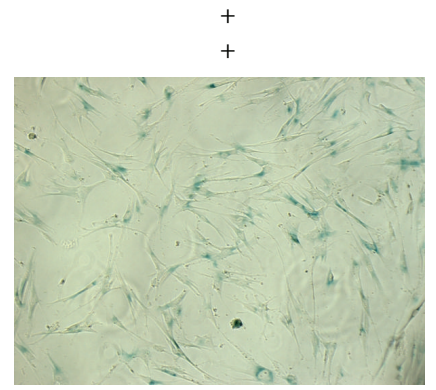

.

(1)

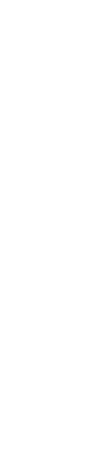

.

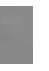

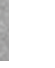 \\ (1)}<smiles>O=C(/C=C/c1ccc(O)cc1)CC(=O)/C=C/c1ccc(O)cc1</smiles>

(d)

FIGURE 2: BDMC attenuated $t$-BHP-induced alterations of senescence-associated features in WI38 cells. WI38 cells at early passage were treated with $20 \mu \mathrm{M}$ BDMC for $48 \mathrm{~h}$, followed by $t$-BHP exposure as described in Materials and Methods to induce premature senescence, and then SA $\beta$-gal staining (a) or DAPI staining (b) was performed. (c) Cells were treated with different dose of BDMC (5-40 $\mu \mathrm{M})$ for $48 \mathrm{~h}$. Then, expressions of $\mathrm{p} 16, \mathrm{p}-\mathrm{Rb}$, and $\mathrm{Rb}$ were examined by Western blot assay. (d) The chemical structure of BDMC.

In the current study, we examined premature senescence of WI38 HDFs after exposure to subcytotoxic dosage of $t$ BHP at $30 \mu \mathrm{M}$ for $5 \mathrm{~d}$ followed by $2 \mathrm{~d}$ recovery according to previous reports [21]. As shown in Figure 1, cells treated with $t$-BHP displayed features resembling replicative senescence as characterized by the enlarged cellular appearance, higher frequency of SA $\beta$-Gal staining, and appearance of SAHF in nuclei when compared to the early passage cells (Figures 1(a) and 1(b)). We also analyzed the effects of $t$ BHP on cell proliferation by MTT assay since a decrease in growth is another well-known feature of cellular senescence. We observed that $t$-BHP treatment decreased the proliferation of HDFs (Figure 1(c)). Furthermore, changes in various senescence-associated molecules were examined at the protein level. Figure 1(d) showed that phosphorylation level of extracellular-signal-regulated kinases 1/2 (ERK1/2), which represents growth factor responsiveness, was decreased in both $t$-BHP-treated and senescent cells. The levels of p16 and $\mathrm{p}-\mathrm{Rb}$, the most well-known molecules in senescence HDFs, were also examined. Increased p16 expression, as well as decreased $\mathrm{Rb}$ phosphorylation, has been reported in senescent HDF cells [19]. Consistently, we observed changes in these proteins in the $t$-BHP-treated cells which were similar to senescent cells. All together, these data suggested that $t$-BHP exposure induced a replicative senescent-like phenotype in WI38 HDFs.

3.2. BDMC Decreased Changes of Senescence-Associated Features in HDFs after $t$-BHP Treatment. To investigate the prevention of $\mathrm{BDMC}$ on oxidative stress-induced premature senescence, HDFs were exposed to $20 \mu \mathrm{M}$ BDMC for $48 \mathrm{~h}$ before stimulation by $t$-BHP. As shown in Figure 2, $t$-BHPtreated WI38 HDFs showed an enlarged and flattened morphology, increased SA $\beta$-Gal positive staining, and SAHF accumulation which were consistent with earlier results. BDMC treatment was shown to markedly protect cells against 

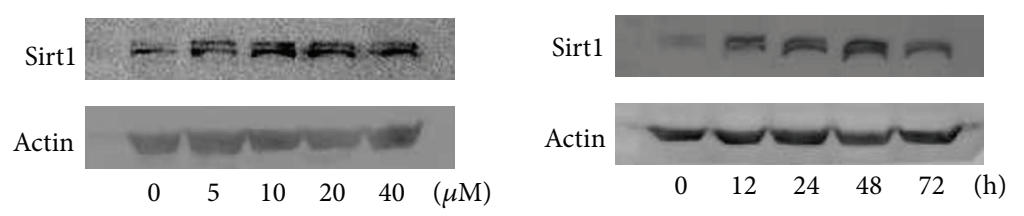

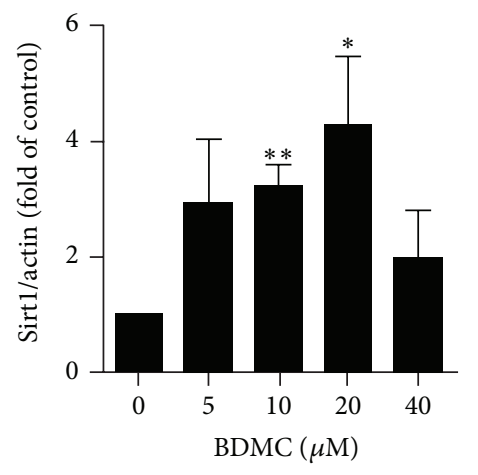

(a)

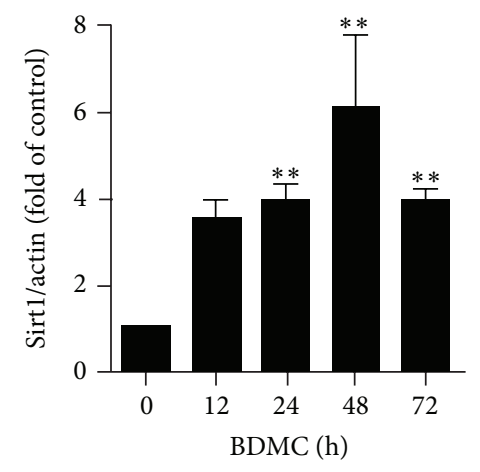

(b)

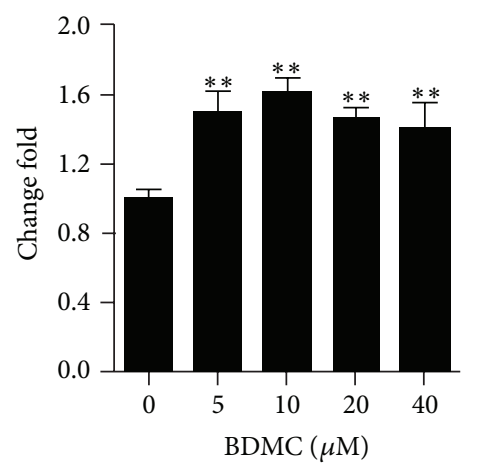

(c)

FIGURE 3: BDMC increased Sirtl in transcriptional and translational levels in WI38 cells. (a) Dose response: cells were treated with different doses of BDMC $(5-40 \mu \mathrm{M})$ for $48 \mathrm{~h}$. (b) Time response: cells were exposed to $20 \mu \mathrm{M}$ of BDMC for different duration (12-72 h). Then Sirtl expression was measured by Western blot assay. (c) Cells were treated with different doses of BDMC (5-40 $\mu \mathrm{M})$ for $24 \mathrm{~h}$; then total RNA was extracted, and reversed transcription, and real-time PCR were performed to assess Sirtl gene level. Data were presented as mean \pm SD of three independent experiments. ${ }^{*} P<0.05 ;{ }^{* *} P<0.01$ compared with control group.

$t$-BHP-induced senescence and shown only sporadic SA $\beta$ Gal positive staining and appearance of SAHF within the nuclei of WI38 cells as well. Furthermore, since the $16 / \mathrm{Rb}$ signaling pathway was indicated to play a crucial role in cellular senescence [24], we examined the effects of BDMC on p16 and its effector, retinoblastoma protein (Rb). HDFs were exposed to different concentrations of BDMC (5$40 \mu \mathrm{M})$, and their effects on the expression and activation of p16 and $\mathrm{Rb}$ were analyzed. It was found that exposure of BDMC decreased p16 protein expression and increased the phosphorylation and total expression of $\mathrm{Rb}$ in a dosedependent manner (Figure 2(c)). These results implied that p16/Rb pathway might be involved in the effect of BDMC in WI38 HDFs.

3.3. BDMC Upregulated Sirt1 Expression in WI38 HDFs. Sirtl is well characterized for its beneficial effects against ageing-related diseases [19, 25-27]. Therefore, we examined whether BDMC could affect Sirtl in WI38 HDFs. As shown in Figure 3(a), treatment with BDMC $(5-40 \mu \mathrm{M})$ for $48 \mathrm{~h}$ increased Sirtl expression. A $12 \mathrm{~h}$ treatment with $20 \mu \mathrm{M}$ BDMC upregulated Sirtl expression, while its expression was highest at $48 \mathrm{~h}$ posttreatment compared to control cells (Figure 3(b)). The upregulation of Sirtl was also confirmed by real-time PCR analysis. The results indicated that Sirt1 mRNA in WI38 HDFs was elevated by BDMC as low as $5 \mu \mathrm{M}$ and displayed an approximately 1.6-fold increase at concentrations of 10 to $40 \mu \mathrm{M}$ (Figure 3(c)). These results indicated that BDMC may exert its effects by modulating Sirt1 expression in WI38 HDFs.
3.4. BDMC Activated AMPK in WI38 HDFs. AMP-activated protein kinase (AMPK), a crucial sensor for alterations in nutrients and intracellular energy levels, can be activated by caloric restriction, which is well accepted to improve agerelated health and retard the ageing process $[25,28,29]$. In addition, it has been reported that Sirtl is a key upstream activator of AMPK signaling [15]. After knowing that Sirt1 was affected by BDMC, we next examined whether BDMC could stimulate AMPK activation. HDFs were treated with different concentrations of BDMC $(5-40 \mu \mathrm{M})$ for $48 \mathrm{~h}$, and then phosphorylation level of AMPK and total AMPK level were examined. As shown in Figure 4(a), BDMC increased the phosphorylation of AMPK without changing the total AMPK level, and the increase reached its maximal at $10 \mu \mathrm{M}$. We next used a pharmacological inhibitor of Sirtl, nicotinamide (NAM), to confirm that activation of AMPK by BDMC was caused by the increase of Sirtl in WI38 HDFs. We observed that NAM-treated HDFs at $40 \mathrm{mM}$ [27], a concentration previously used to significantly decreased Sirtl level, as well as block the activation of AMPK induced by BDMC (Figure 4(b)). These results suggested that AMPK stimulation by BDMC was mediated by Sirtl increase.

3.5. Protective Effects against $t$-BHP-Induced Senescence Are Partially Associated with the Alteration of Sirt1. To further explore the correlation between the protection of BDMC against $t$-BHP-induced premature senescence and the alteration in Sirtl levels, we used the pharmacological inhibitor NAM or siRNA to decrease Sirtl expression in WI38 HDFs. After transfection of Sirtl siRNA or NAM treatment, WI38 


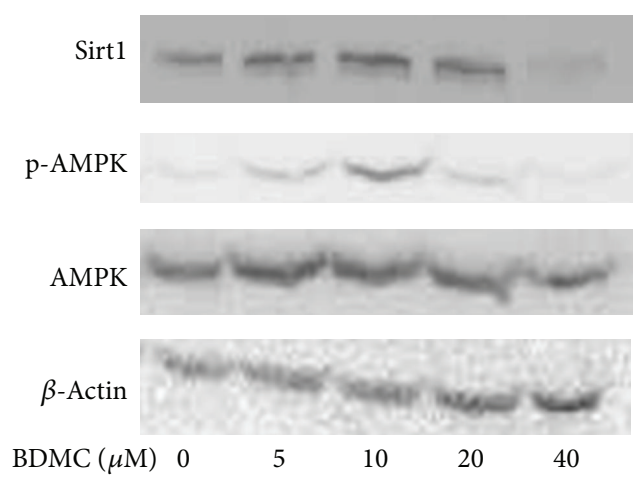

(a)

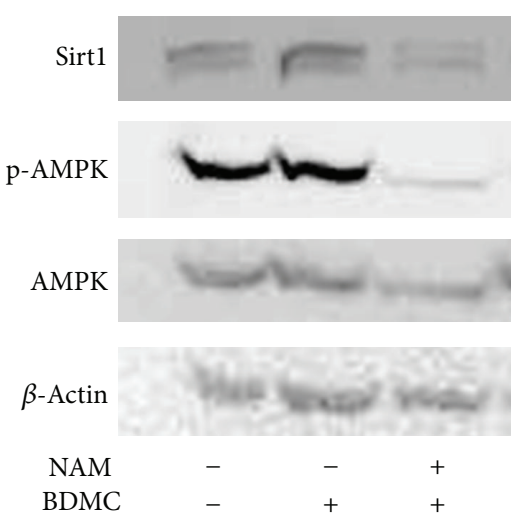

(b)

FIGURE 4: BDMC activated AMPK with the increase in Sirt1. (a) WI38 cells were treated with different doses of BDMC (5-40 $\mu \mathrm{M})$ for $48 \mathrm{~h}$. Then, phosphorylation level of AMPK and total AMPK protein level were measured by Western blot assay. (b) WI38 cells were treated with nicotinamide (NAM) for 1-2 h, followed by BDMC $(20 \mu \mathrm{M})$ for $48 \mathrm{~h}$; then Sirtl, p-AMPK, and AMPK were examined by Western blot assay.

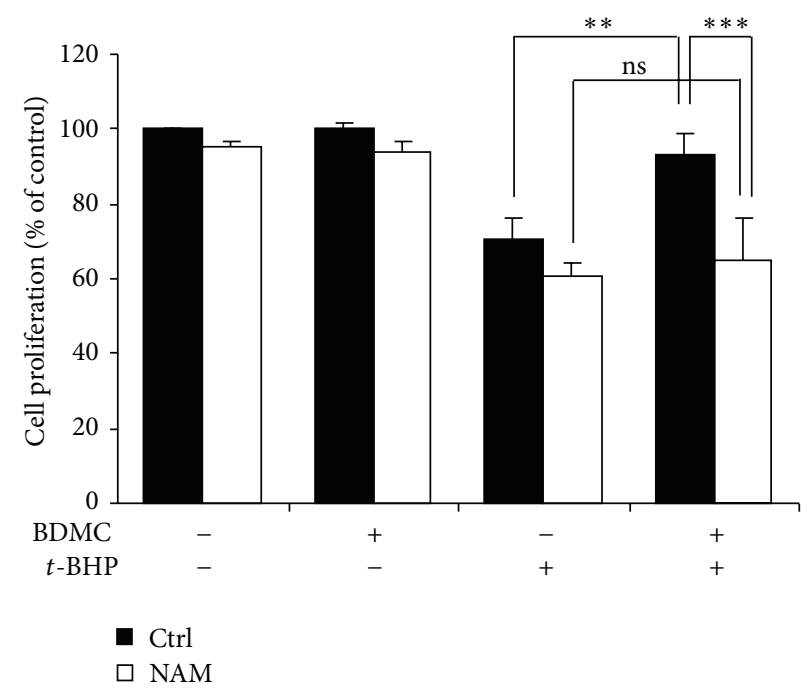

(a)

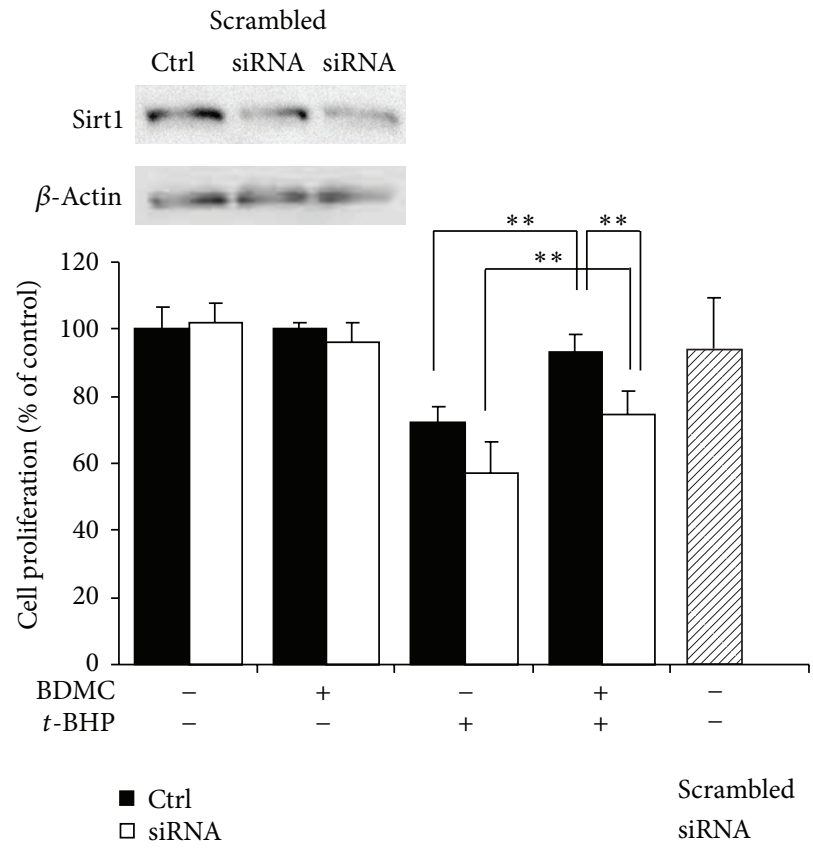

(b)

FIGURE 5: Inhibition in Sirtl partially compromised the beneficial effects of BDMC on $t$-BHP-induced cellular senescence in WI38 cells. WI38 cells were pretreated with NAM $(40 \mathrm{mM})$ for $1 \mathrm{~h}$ (a), or transfected with Sirtl siRNA (b), then incubated in the presence or absence of BDMC $(20 \mu \mathrm{M})$ for $48 \mathrm{~h}$, followed by exposure to $t$-BHP treatment. Cell proliferation was then determined by MTT assay. Data were presented as mean $\pm \mathrm{SD}$ of three independent experiments. ${ }^{*} P<0.05 ;{ }^{* *} P<0.01$.

HDFs were treated in the presence or absence of $20 \mu \mathrm{M}$ BDMC for $48 \mathrm{~h}$, followed by stimulation by $t$-BHP. MTT assay was performed to assess cell proliferation. As shown in Figure 5, transfection with Sirtl siRNA and pretreatment with NAM decreased Sirtl protein levels with no cytotoxic effects observed when compared to the control.

Cell proliferation was reduced to $77.7 \pm 9.8 \%$ of the control after incubating with $t$-BHP. Cell proliferation was significantly reduced to $64.5 \pm 7.8 \%$ and $57.3 \pm 9.0 \%$ after treatment with $t$-BHP in siRNA-transfected or NAM-treated cells, respectively, compared to $t$-BHP-treated cells alone. This suggested that Sirtl may protect against oxidative insults. Moreover, BDMC treatment attenuated $t$-BHP-induced loss of cell proliferation capability. However, this protection was partly compromised by the decrease of Sirtl by either siRNA or NAM, suggesting that BDMC prevented the loss of 
proliferation via Sirtl increase. However, other mechanisms may contribute to the effects of BDMC since BDMC still exerted slight protection in Sirtl knockdown group.

\section{Discussion}

Human diploid fibroblast cells can permanently lose their proliferative potential after a limited number of population doublings and enter replicative senescence [30]. Numerous lines of evidence have suggested that exposure to exotic oxidative stress can trigger stress-induced premature senescence in HDFs within a few days. This premature senescence resulted from accumulation of random damage and loss of repair function caused by reactive oxygen species (ROS), and shared common features with replicative senescence [31]. In this study, we analyzed the long-term effects of sublethal oxidative insults using $t$-BHP in WI38 HDFs. In line with previous reports, treatment with $t$-BHP induced senescence of HDFs, indicated by an enlarged cellular appearance, a higher frequency of SA $\beta$-Gal staining activity, accumulation of SAFH in nucleus, and changes in the molecular markers including p16 and $\mathrm{Rb}$.

Data obtained from laboratory experiments and clinical trials showed the potential of BDMC to improve ageassociated disorders. BDMC has been reported to protect endothelial cells against $t$-BHP-induced cell damage [32], by inducing hemo oxygenase-1 expression through decreasing nitric oxide synthase expression and nitric oxide production [33]. In addition, study in patients with sporadic Alzheimer's disease showed that BDMC could improve the innate immune system and increase amyloid-beta clearance from the brain [34]. In this study, we examined the effects of BDMC on oxidative stress-induced senescence in WI38 HDFs. Our results indicated that pretreatment with $20 \mu \mathrm{M}$ BDMC could attenuate $t$-BHP-induced changes of HDFs, including decrease in SA $\beta$-Gal positive staining and appearance of SAHF. Most of the reports have attributed the protection of curcuminoids to their maintenance on cellular redox homeostasis. On one hand, curcuminoids are well known for their promising antioxidative effects due to the phenolic hydroxyl group in their molecular structure. On the other hand, it has been demonstrated that curcuminoids could induce the expression of antioxidant enzymes such as superoxide dismutase and heme oxygenase- 1 and thereby exert cytoprotective effects in response to oxidative insults. It is interesting to find in our study that BDMC dose dependently regulated $\mathrm{p} 16 / \mathrm{Rb}$, one of the major senescence-related pathways, suggesting that ROS-irrelevant mechanisms may also contribute to the protective effects of BDMC against oxidative insults.

Much interest has been shown of Sirtl due to its essential role in metabolism, longevity, and stress response. It has been demonstrated that caloric restriction retards ageing process by modulating Sirtl function in many species ranging from yeast to primates. An increase in Sirtl protein by genetic methods or pharmacological activators can extend the lifespan of organisms and prevent cellular senescence $[8,16,24,35]$. Our data in Figure 1(d) showed that, similar to the cells of late passage, Sirt1 level was also decreased in $t$-BHP-induced premature senescent cells as compared with cells of early passage, suggesting that this protein may take part in the oxidative stress-induced premature senescence. In addition, it was reported that some polyphenols conferred protection against oxidative stress-induced cell apoptosis and senescence through activation of Sirt1 $[10,36]$. Therefore, we hypothesized that Sirtl might also be involved in the protective effects of BDMC against $t$-BHP-induced premature senescence in HDFs. Our results demonstrated that both Sirtl protein and mRNA level increased upon treatment with BDMC. Meanwhile, AMPK, which is a sensor of cellular energy status, was also up regulated by BDMC with the increase of Sirtl. This observation is consistent with previous reports that demonstrated curcuminoids increased AMPK phosphorylation and its downstream factor acetylCoA carboxylase in hepatoma cells [37]. Additionally, AMPK activation has been shown to regulate curcumin-stimulated glucose uptake in myotube cells [38]. These data raised the possibility that curcumin-activated AMPK is involved in cell metabolism and thereby may be related to BDMC-increased Sirt1. Recent studies in hepatocytes indicated that activation of Sirtl stimulated the basal AMPK signaling pathways and prevented lipid accumulation $[15,39]$, suggesting that AMPK is an important downstream target of Sirtl. Additionally, AMPK activation was suggested to be a crucial mechanism for polyphenol-mediated effects $[15,40]$. Therefore, we explored whether BDMC activated AMPK in a Sirtldependent manner. For this, a Sirtl inhibitor NAM was used in the study. The results showed that treatment with BDMC led to an increase of p-AMPK after $48 \mathrm{~h}$ treatment. However, this increase could not be observed in the NAM pretreatment cells, indicating that BDMC-induced AMPK activation was dependent on Sirtl.

We further explored whether the beneficial effect of BDMC against $t$-BHP-induced premature senescence was through the increase of Sirtl. Sirtl protein in WI38 HDFs was inhibited by siRNA or NAM, and then these cells were incubated with BDMC followed by $t$-BHP-induced senescence. Consistent with previous reports, our results demonstrated that cells with decreased level of Sirtl were more sensitive to $t$-BHP-induced damage than normal cells, indicating that $S i r t 1$ plays a role in protecting against oxidative insults and decreased growth. Moreover, we found that the protective effects of BDMC were partly blocked in cells with decreased level of Sirtl. This observation provided strong support that BDMC exerted its preventive effects on $t$ BHP-induced senescence-like growth arrest by increasing Sirtl. However, although this protective effect was attenuated after downregulation in Sirtl, BDMC still exhibited partial protection to WI38 cells. This may be due to residual Sirt1 level that was not completely abolished by the pharmacological inhibitor or siRNA. Furthermore, this suggests that other mechanisms may be involved, which contribute to the protective effects of BDMC. Further studies are needed to identify additional mechanisms, such as the enhancement of antioxidant defenses.

Overall, our study indicated that BDMC prevented $t$ BHP-induced senescence-like growth arrest in WI38 HDFs. 
We further showed that this might be dependent on the increase of Sirtl and subsequent activation of AMPK. This is to our knowledge the first report linking Sirtl with the protective effects of BDMC against oxidative-stress-induced cellular senescence in WI38 fibroblast cells. Although other mechanisms, such as antioxidative pathways, could not be excluded, the link between BDMC and Sirtl provides novel insight into the therapeutic potential of BDMC as well as curcuminoids. Apart from the important role in longevity, Sirtl/AMPK has also been regarded as a crucial link between cell metabolism and stress response. Thus, this study also provides possibility that regulation on Sirtl could be the underlying mechanisms of other beneficial effects of curcuminoids.

\begin{tabular}{|c|c|}
\hline \multicolumn{2}{|c|}{ Abbreviations } \\
\hline BDMC: & Bisdemethoxycurcumin \\
\hline HDF: & Human diploid fibroblast \\
\hline SA- $\beta$-Gal: & Senescence-associated $\beta$-Gal \\
\hline SAHF: & $\begin{array}{l}\text { Senescence-associated heterochromatic } \\
\text { foci }\end{array}$ \\
\hline ROS: & Reactive oxygen species \\
\hline BME: & Basal medium Eagle medium \\
\hline FCS: & Fetal calf serum \\
\hline DAPI: & $4^{\prime}$-6-Diamidino-2-phenylindole \\
\hline MTT: & $\begin{array}{l}\text { 3-(4,5-Dimethyl-2-thiazolyl)-2,5- } \\
\text { diphenyl tetrazolium } \\
\text { bromide }\end{array}$ \\
\hline $\mathrm{Rb}:$ & Retinoblastoma protein \\
\hline ERK: & Extracellular-regulated protein kinases. \\
\hline
\end{tabular}

\section{Conflict of Interests}

The authors have declared no conflict of interests.

\section{Acknowledgments}

The current study was supported by Macao Science and Technology Development Fund (074/2012/A3 and 077/2011/A3) and the Research Fund of University of Macau (MYRG208 (Y1-L4)-ICMS11-WYT and RG084/09-10S/HPM/ICMS).

\section{References}

[1] B. L. Queen and T. O. Tollefsbol, "Polyphenols and aging," Current Aging Science, vol. 3, no. 1, pp. 34-42, 2010.

[2] A. Goel, A. B. Kunnumakkara, and B. B. Aggarwal, "Curcumin as "Curecumin": from kitchen to clinic," Biochemical Pharmacology, vol. 75, no. 4, pp. 787-809, 2008.

[3] T. Esatbeyoglu, P. Huebbe, I. M. Ernst et al., "Curcuminfrom molecule to biological function," Angewandte Chemie International Edition, vol. 51, no. 22, pp. 5308-5332.

[4] G. Mao, Y. Wang, Q. Qiu et al., "Salidroside protects human fibroblast cells from premature senescence induced by $\mathrm{H}_{2} \mathrm{O}_{2}$ partly through modulating oxidative status," Mechanisms of Ageing and Development, vol. 131, no. 11-12, pp. 723-731, 2010.

[5] V. Calabrese, T. E. Bates, C. Mancuso et al., "Curcumin and the cellular stress response in free radical-related diseases,"
Molecular Nutrition and Food Research, vol. 52, no. 9, pp. 10621073, 2008

[6] K. Lee, B. Lee, S. Semnani et al., "Curcumin extends life span, improves health span, and modulates the expression of age-associated aging genes in drosophila melanogaster," Rejuvenation Research, vol. 13, no. 5, pp. 561-570, 2010.

[7] K. Kitani, T. Osawa, and T. Yokozawa, "The effects of tetrahydrocurcumin and green tea polyphenol on the survival of male C57BL/6 mice," Biogerontology, vol. 8, no. 5, pp. 567-573, 2007.

[8] Y. Zu, L. Liu, M. Y. K. Lee et al., "SIRT1 promotes proliferation and prevents senescence through targeting LKB1 in primary porcine aortic endothelial cells," Circulation Research, vol. 106, no. 8, pp. 1384-1393, 2010.

[9] W. Yu, Y. Fu, X. Zhou et al., "Effects of resveratrol on $\mathrm{H}_{2} \mathrm{O}_{2}$ induced apoptosis and expression of SIRTs in H9c2 cells," Journal of Cellular Biochemistry, vol. 107, no. 4, pp. 741-747, 2009.

[10] C. Kao, L. Chen, Y. Chang et al., "Resveratrol protects human endothelium from $\mathrm{H}_{2} \mathrm{O}_{2}$-induced oxidative stress and senescence via SirT1 activation," Journal of Atherosclerosis and Thrombosis, vol. 17, no. 9, pp. 970-979, 2010.

[11] V. Parashar and B. Rogina, "dSir2 mediates the increased spontaneous physical activity in flies on calorie restriction," Aging, vol. 1, no. 6, pp. 529-541, 2009.

[12] K. Zarse, S. Schmeisser, M. Birringer, E. Falk, D. Schmoll, and M. Ristow, "Differential effects of resveratrol and SRT1720 on lifespan of adult caenorhabditis elegans," Hormone and Metabolic Research, vol. 42, no. 12, pp. 837-839, 2010.

[13] M. A. Wilson, B. Shukitt-Hale, W. Kalt, D. K. Ingram, J. A. Joseph, and C. A. Wolkow, "Blueberry polyphenols increase lifespan and thermotolerance in Caenorhabditis elegans," Aging Cell, vol. 5, no. 1, pp. 59-68, 2006.

[14] A. Kampkötter, C. Timpel, R. F. Zurawski et al., "Increase of stress resistance and lifespan of Caenorhabditis elegans by quercetin," Comparative Biochemistry and Physiology B, vol. 149, no. 2, pp. 314-323, 2008.

[15] X. Hou, S. Xu, K. A. Maitland-Toolan et al., "SIRT1 regulates hepatocyte lipid metabolism through activating AMP-activated protein kinase," The Journal of Biological Chemistry, vol. 283, no. 29, pp. 20015-20026, 2008.

[16] S. Imai, "Is Sirtl a miracle bullet for longevity?" Aging Cell, vol. 6, no. 6, pp. 735-737, 2007.

[17] V. D. Longo and B. K. Kennedy, "Sirtuins in aging and agerelated disease," Cell, vol. 126, no. 2, pp. 257-268, 2006.

[18] G. P. Dimri, X. Lee, G. Basile et al., "A biomarker that identifies senescent human cells in culture and in aging skin in vivo," Proceedings of the National Academy of Sciences of the United States of America, vol. 92, no. 20, pp. 9363-9367, 1995.

[19] J. Huang, Q. Gan, L. Han et al., "SIRT1 overexpression antagonizes cellular senescence with activated ERK/S6k1 signaling in human diploid fibroblasts," PLoS ONE, vol. 3, no. 3, Article ID e1710, 2008.

[20] Q. M. Chen, J. C. Bartholomew, J. Campisi, M. Acosta, J. D. Reagan, and B. N. Ames, "Molecular analysis of $\mathrm{H}_{2} \mathrm{O}_{2}$-induced senescent-like growth arrest in normal human fibroblasts: p53 and Rb control G1 arrest but not cell replication," The Biochemical journal, vol. 332, part 1, pp. 43-50, 1998.

[21] P. Dumont, M. Burton, Q. M. Chen et al., "Induction of replicative senescence biomarkers by sublethal oxidative stresses in normal human fibroblast," Free Radical Biology and Medicine, vol. 28, no. 3, pp. 361-373, 2000. 
[22] P. Dumont, F. Chainiaux, F. Eliaers et al., "Overexpression of apolipoprotein J in human fibroblasts protects against cytotoxicity and premature senescence induced by ethanol and tertbutylhydroperoxide," Cell Stress and Chaperones, vol. 7, no. 1, pp. 23-35.

[23] J. Dierick, D. E. Kalume, F. Wenders et al., "Identification of 30 protein species involved in replicative senescence and stressinduced premature senescence," FEBS Letters, vol. 531, no. 3, pp. 499-504, 2002.

[24] J. Duan, Z. Zhang, and T. Tong, "Senescence delay of human diploid fibroblast induced by anti-sense p16 INK4a expression," The Journal of Biological Chemistry, vol. 276, no. 51, pp. 4832548331, 2001.

[25] V. N. Anisimov, "Premature ageing prevention: limitations and perspectives of pharmacological interventions," Current Drug Targets, vol. 7, no. 11, pp. 1485-1503, 2006.

[26] C. L. Brooks and W. Gu, "How does SIRT1 affect metabolism, senescence and cancer?" Nature Reviews Cancer, vol. 9, no. 2, pp. 123-128, 2009.

[27] C. Chen, W. Yu, Y. Fu, X. Wang, J. Li, and W. Wang, "Resveratrol protects cardiomyocytes from hypoxia-induced apoptosis through the SIRT1-FoxO1 pathway," Biochemical and Biophysical Research Communications, vol. 378, no. 3, pp. 389393, 2009.

[28] K. Kajita, T. Mune, T. Ikeda et al., "Effect of fasting on PPAR $\gamma$ and AMPK activity in adipocytes," Diabetes Research and Clinical Practice, vol. 81, no. 2, pp. 144-149, 2008.

[29] A. E. Carrillo and A. D. Flouris, "Caloric restriction and longevity: effects of reduced body temperature," Ageing Research Reviews, vol. 10, no. 1, pp. 153-162, 2011.

[30] L. Hayflick, "The limited in vitro lifetime of human diploid cell strains," Experimental Cell Research, vol. 37, no. 3, pp. 614-636, 1965.

[31] O. Toussaint, E. E. Medrano, and T. Von Zglinicki, "Cellular and molecular mechanisms of stress-induced premature senescence (SIPS) of human diploid fibroblasts and melanocytes," Experimental Gerontology, vol. 35, no. 8, pp. 927-945, 2000.

[32] Y. Li, J. Gao, S. M. Lee, Q. Zhang, P. Hoi, and Y. Wang, "Bisdemethoxycurcumin protects endothelial cells against $t$ BHP-induced cell damage by regulating the phosphorylation level of ERK1/2 and Akt," International Journal of Molecular Medicine, vol. 27, no. 2, pp. 205-211, 2011.

[33] A. N. Kim, W. Jeon, J. J. Lee, and B. Kim, "Up-regulation of heme oxygenase-1 expression through CaMKII-ERK1/2-Nrf2 signaling mediates the anti-inflammatory effect of bisdemethoxycurcumin in LPS-stimulated macrophages," Free Radical Biology and Medicine, vol. 49, no. 3, pp. 323-331, 2010.

[34] M. Fiala, P. T. Liu, A. Espinosa-Jeffrey et al., "Innate immunity and transcription of MGAT-III and Toll-like receptors in Alzheimer's disease patients are improved by bisdemethoxycurcumin," Proceedings of the National Academy of Sciences of the United States of America, vol. 104, no. 31, pp. 12849-12854, 2007.

[35] J. G. Wood, B. Rogina, S. Lavu et al., "Sirtuin activators mimic caloric restriction and delay ageing in metazoans," Nature, vol. 430, no. 7000, pp. 686-689.

[36] H. Ota, M. Eto, M. R. Kano et al., "Cilostazol inhibits oxidative stress-induced premature senescence via upregulation of Sirtl in human endothelial cells," Arteriosclerosis, Thrombosis, and Vascular Biology, vol. 28, no. 9, pp. 1634-1639, 2008.

[37] T. Kim, J. Davis, A. J. Zhang, X. He, and S. T. Mathews, "Curcumin activates AMPK and suppresses gluconeogenic gene expression in hepatoma cells," Biochemical and Biophysical Research Communications, vol. 388, no. 2, pp. 377-382, 2009.

[38] J. H. Kim, J. M. Park, E. Kim et al., "Curcumin stimulates glucose uptake through AMPK-p38 MAPK pathways in L6 myotube cells," Journal of Cellular Physiology, vol. 223, no. 3, pp. 771-778, 2010.

[39] G. Suchankova, L. E. Nelson, Z. Gerhart-Hines et al., "Concurrent regulation of AMP-activated protein kinase and SIRT1 in mammalian cells," Biochemical and Biophysical Research Communications, vol. 378, no. 4, pp. 836-841, 2009.

[40] G. R. Steinberg and B. E. Kemp, "AMPK in health and disease," Physiological Reviews, vol. 89, no. 3, pp. 1025-1078, 2009. 


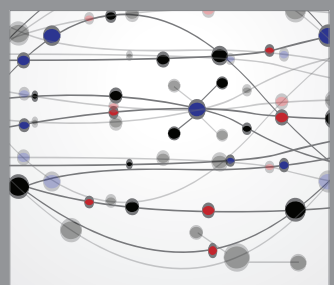

The Scientific World Journal
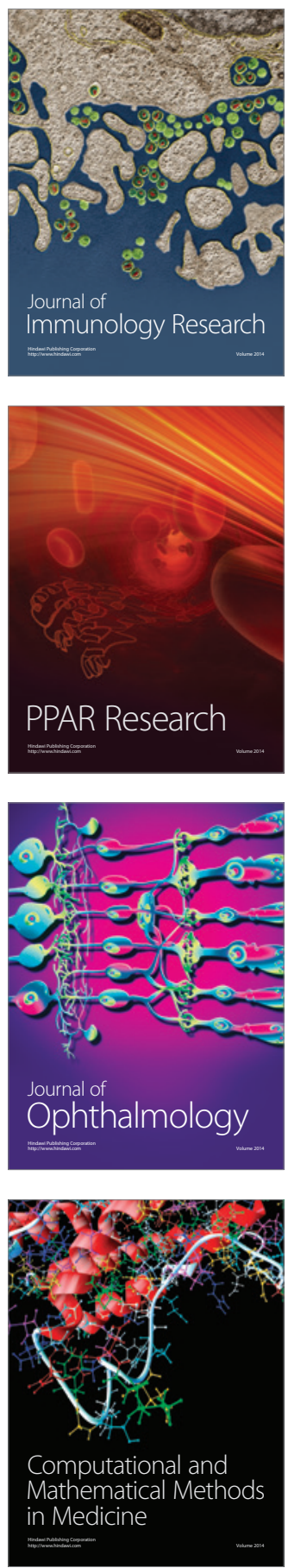

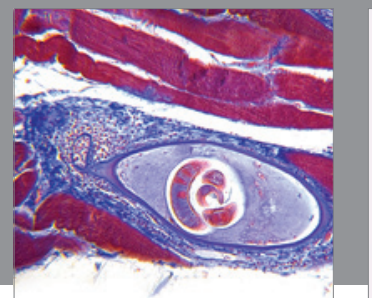

Gastroenterology

Research and Practice
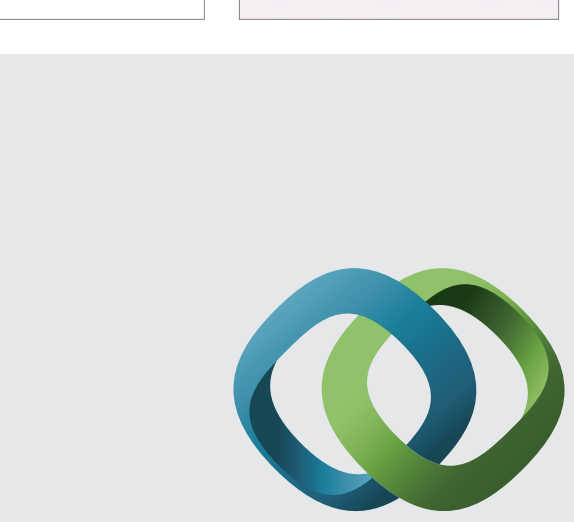

\section{Hindawi}

Submit your manuscripts at

http://www.hindawi.com
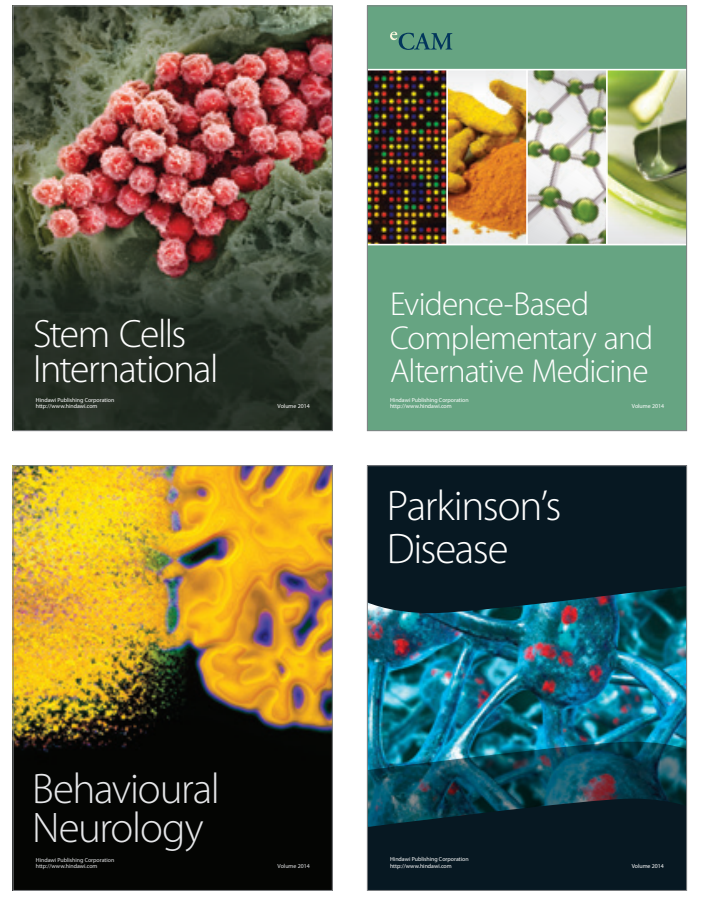
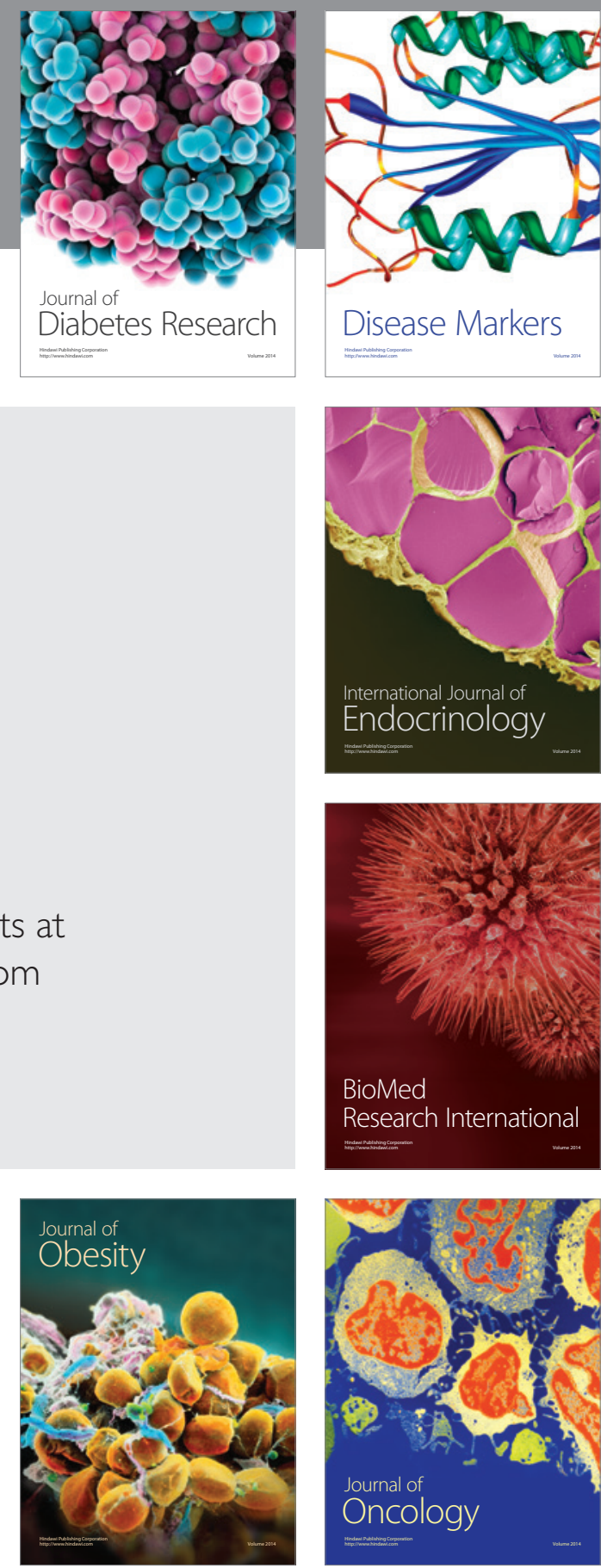

Disease Markers
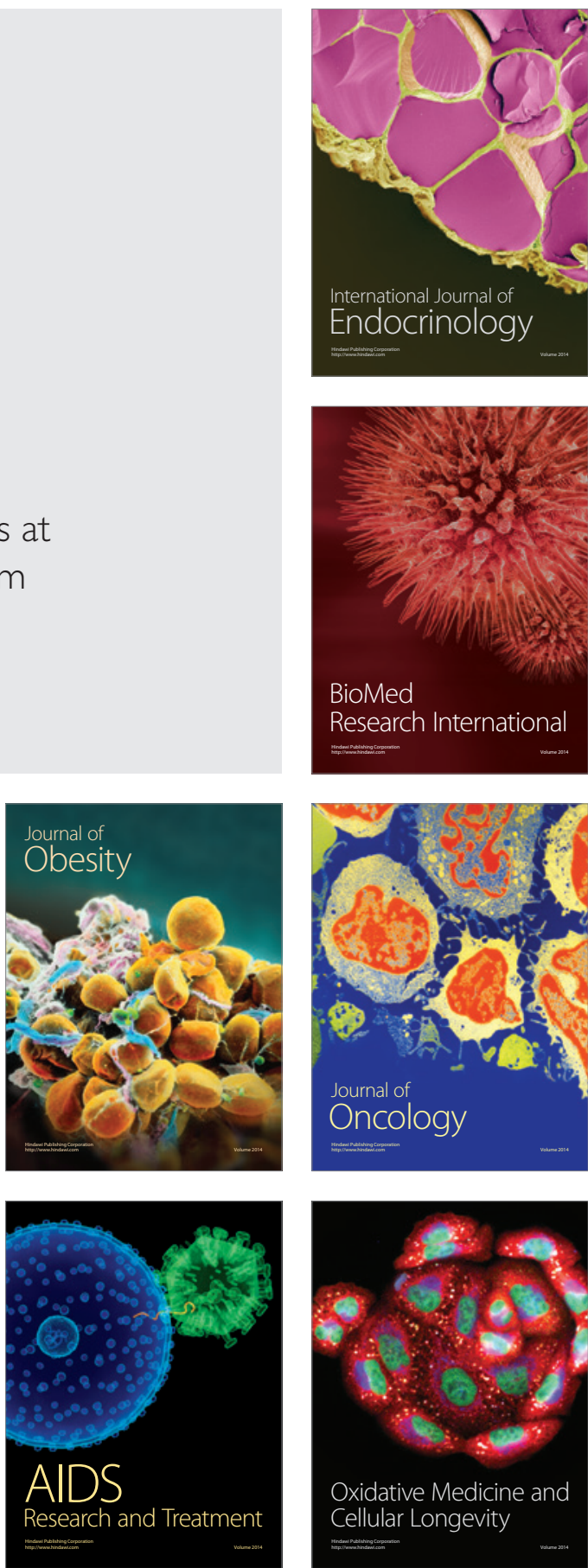